\title{
A Mechanism For The Elimination Of The Female Gamete Centrosome
}

Pimenta-Marques A. ${ }^{1, \#}$, Bento I. $^{1,2, \#}$, Lopes C.A.M. ${ }^{1,}$, Duarte P. ${ }^{1}$, Jana S.C. ${ }^{1}$, BettencourtDias M. ${ }^{1}$

\# equal contribution

${ }^{1}$ Instituto Gulbenkian de Ciência, Rua da Quinta Grande, 2780-156 Oeiras, Portugal

2 Present Address: Instituto de Medicina Molecular, Avenida Professor Egas Moniz, 1649028 Lisboa, Portugal

To whom correspondence should be addressed:

mdias@igc.gulbenkian.pt

armarques@igc.gulbenkian.pt

inesbento@hotmail.com 


\section{Abstract}

An important feature of fertilization is the asymmetric inheritance of centrioles. In most species it is the sperm that contributes the initial centriole, which builds the first centrosome that is essential for early development. However, given that centrioles are thought to be exceptionally stable structures, the mechanism behind centriole disappearance in the female germline remains elusive and paradoxical. Here, we show a general program for centriole maintenance, led by Polo kinase and the pericentriolar matrix (PCM), which is down-regulated in the female germline, resulting in centriole loss. Perturbing this program prevents centriole loss, leading to abnormal meiotic and mitotic divisions, and associated female sterility. This mechanism challenges the view that centrioles are intrinsically stable structures and shows novel general functions for Polo kinase and the PCM in centriole maintenance. We propose that regulation of this maintenance program is essential for successful sexual reproduction, and defines centriole life span in different tissues in homeostasis and disease, shaping the cytoskeleton. 


\section{INTRODUCTION}

Some organelles are asymmetrically inherited upon fertilization, as it is the case for mitochondria, which are provided maternally, while the paternal complement is actively degraded by autophagy (1). Centrosomes, the major microtubule-organizing centers (MTOC) in animal cells, are composed by two very stable microtubule (MT) cylinders, the centrioles, and a pericentriolar protein matrix (PCM). The PCM is indispensable for centriole biogenesis and centrosome function to nucleate and anchor MTs (2). The number of centrioles, and consequently the number of centrosomes, is tightly controlled in cycling cells: centriole duplication is coupled to DNA replication so that in mitosis there is one centrosome with two centrioles at each pole of the mitotic spindle, ensuring faithful chromosome segregation. Surprisingly, centrioles are eliminated in the oocytes of most metazoan species and the embryo relies on the centriole provided by the sperm, which subsequently duplicates and forms two centrosomes supporting successful mitotic divisions (3-6). Centriole elimination in the egg is commonly regarded as a strategy to ensure correct centriole number upon fertilization, preventing abnormal first embryonic mitosis. Moreover, it is also thought to prevent parthenogenesis, since the microinjection of centrioles in frog eggs induces successful embryonic development without fertilization (7).

Two different timing and strategies for centriole elimination occur in the female germline of different animals. In mollusks and echinoderms, centrosomes with centrioles are present in meiosis and a mechanism has been elucidated recently in starfish, where most centrioles are eliminated by extrusion in the polar bodies $(4,8)$. In contrast, in fruit flies, worms and humans, centrioles are eliminated before meiotic division, one of the few acentriolar divisions in those species $(3,4,6,9)$. Despite the fact that centriole elimination before meiotic division is such a widespread event, little is known concerning the molecular mechanism governing this process. Additionally, the real consequences of retaining centrioles, for oogenesis progression, meiosis and reproduction, are still unclear. Here, we used Drosophila melanogaster oogenesis to identify the molecular mechanisms of centriole elimination and the consequences of preventing it. 


\section{RESULTS}

\section{Centrioles are eliminated in late oogenesis}

Huettner first pointed out in 1933 (10) the acentriolar nature of Drosophila female meiotic division, intensifying the discussion among cell biology pioneers on centriole inheritance, a problem he considered "very intricate and perplexing". Almost half a century later, Mahowald (11) beautifully showed by electron microscopy (EM) the complex behavior of centrioles during Drosophila oogenesis. Oogenesis begins with the asymmetric division of a stem cell to give rise to a new stem cell and a cystoblast, which undergoes 4 successive mitotic cycles with incomplete cytokinesis, forming a large cyst composed of 16 interconnected cells. Mahowald (11) detected up to 25 identifiable mature centrioles and additional procentrioles in the entire cyst (procentrioles were also seen by Adelaide Carpenter, personal communication). Those results suggest that centrioles in the 16-cell cyst duplicate, predicting a total count of 64 centrioles and procentrioles in one cyst. One of the 16 cells becomes the oocyte, while the others are called nurse cells (Fig. 1A). Strikingly, early in oogenesis, most centrioles from each one of the 15 nurse cells migrate to the oocyte and cluster, forming a very large MTOC that organizes the MT cytoskeleton from stages 2 to 6 (11) (Fig. 1A), and can be detected up until stage 9 by EM (12).

To investigate when centrioles are eliminated we initially divided oogenesis in 3 easily recognizable stages: early (germarium (G) to stage 6), mid (stages 7 and 8, when the oocyte is repolarized) and late (stages 9 to 12) (Fig. 1A). We first examined the oocyte for the presence of a conserved centriole-specific protein, ANA1, expressed under the control of its endogenous promoter and fused to tdTomato (13) (Fig. 1B,C). We observed centrioles at the beginning of late stages (stage 9), confirming previous studies $(12,14)$. However, we were surprised to detect centrioles later than that, in all oocytes from stage 9 to 12 (Fig. 1C,E). In accordance with published EM studies, in early stages, we observed centrioles clustered at the posterior end of the oocyte between the nucleus and the follicular cell border, mostly as a single uniform very large structure (11). In late stages, ANA1 was present in either one or very few discrete large dots in the nucleus vicinity (Fig. $1 C)$, suggesting centrioles are also clustered. 
We next investigated the presence of centrioles upon nuclear envelope breakdown at the spindle of meiosis I (stage 14). Confirming Huettner's light microscopy pioneering studies (1933) (10), we were unable to detect centrioles at the poles of the spindle (Fig. 1F). We thus conclude that centrioles are present until later stages than previously described (12), but disappear just before meiotic spindle assembly.

\section{Centrosome disassembly is a stepwise process: PCM first, centrioles last}

We reasoned that centrosome elimination could occur in different ways: i) abruptly, with all the structure being lost simultaneously, or ii) the centrosome could disassemble progressively throughout oogenesis. To answer this question we investigated the presence of several centrosome components, i.e. centriole and PCM constituents (Fig. 1B), along oogenesis. We first tested the centriolar components SAS6 and BLD10/CEP135 (Fig. 1B), which are part of the cartwheel, a hallmark structure of the centriole that helps defining its nine-fold symmetry (2). While SAS6 presence until late stages corroborated ANA1 observations, we found a decrease in the number of oocytes containing BLD10, suggesting centrioles start to lose components at those stages (fig. S1A; Fig. 1D,E). We then checked the presence of different PCM constituents (Fig. 1B): i) $\gamma$-tubulin, which is very important for MT nucleation; ii) PCM components that recruit $\gamma$-tubulin (DPLP/Pericentrin, CNN/CDK5RAP2, SPD2/CEP192) (14-18), and iii) peripheral centriole components that recruit the PCM (SAS4/CPAP and ASL/CEP152) $(19,20)$. In early stages all PCM components were present at the centrosomes (Fig. 1G-I; fig. S1B-D). However, contrary to centriolar components, in mid stages some PCM components started to disappear and their loss was aggravated in later stages, with SPD2 loss being particularly evident (only $33 \%$ of the oocytes had SPD2 in mid stages and $8 \%$ in late stages; Fig. $1 \mathrm{H}, \mathrm{I}$ ). $\gamma$-tubulin, which can be recruited by several PCM components (21), was the most stable PCM constituent ( $77 \%$ of late stage oocytes were positive; Fig. $1 G, I)$. Our data suggests that PCM and centriole loss occurs progressively.

We then characterized in more detail the mid and late stages of this process, when the centrosome starts losing some of its components. Because centrioles are very small structures and are densely packed at the late stages of oogenesis, we quantified the total signal for what we observed to be the most stable centriole marker, ANA1 (Fig. 1E), as a proxy for total centriole mass (Fig. 1J). We observed similar total intensities of ANA1- 
tdTomato per oocyte in mid and beginning of late stages (stage 10), and this was roughly 50 times higher than that of single centrioles encountered in very early stages (Fig. 1J). Our experimental approximations to centriole number support the initial calculations and assumptions, based on Mahowald's studies, that most centrioles from the 16 cells duplicate in the germarium and migrate to the oocyte (64 expected centrioles; (11)). The similarity in intensity found in mid stages and stage 10 suggests there is little centriole breakdown at that time in oogenesis. In contrast, in stages 12 and 13, the intensity of ANA1 was much lower, similar to the intensity of 6 "early" centrioles (Fig. 1J). Finally, when we investigated stage 14 (meiotic division; Fig. 1F,J) we only observed two out of twenty eggs showing one or two cortex-localized, non MT-nucleating centrioles (fig. S1E), supporting nearly complete centriole elimination at that stage. We thus conclude that the centrosome disassembles progressively: it starts losing several PCM components in mid stages and this is then followed by the loss of centriolar components in late stages (steeper between stage 10 and 12) leading to complete centriole loss at the meiotic division (stage 14).

\section{Loss of PCM leads to centriole elimination in Drosophila somatic cells}

Our data suggests that centriole elimination could be a consequence of PCM loss. Although little is known about the regulation of the PCM in stable interphasic centrosomes, it is described that most centrioles in this phase are coated by at least one PCM component, in all organisms (22), which might contribute for its stability. Interestingly, Tetrahymena centrioles (called basal bodies) are unstable upon depletion of $\gamma$-tubulin (23), further suggesting a role for the PCM in centriole stability. While a role for the PCM in centriole biogenesis has been acknowledged (2), we hypothesized that the PCM also has an important function in centriole maintenance. To test this hypothesis we developed a 'Centriole Stability Assay' in Drosophila cultured cells. This assay allows to: i) simultaneously deplete several PCM components, circumventing the absence of phenotypes resulting from their known redundant roles, and ii) uncouple centrosome maintenance from centrosome biogenesis. We thus arrested Drosophila tissue culture cells (DMEL) in S-phase (fig. S2A) to halt the centriole biogenesis cycle after centriole duplication (20). Drosophila centrioles are coated by a thin PCM layer, closely associated with centrioles in interphase, and composed of components such as ASL, D-PLP and SPD2, 
and additionally by CNN in G2 (24). We asked whether centrioles were destabilized upon single- or co-depletion of ASL, D-PLP, SPD2 and CNN ('All PCM') for four days (fig. S2A-E). We observed a significant decrease in centriole number (higher percentage of cells with the abnormally low number of 0-1 centrioles) when 'All PCM' was depleted, rather than individual depletion of different PCM components (fig. S2C). These results show that the PCM is required for centriole stability in somatic cells and that PCM components are redundant in that role.

\section{Loss of Polo from the oocyte centrosome co-occurs with PCM loss}

Our results strongly suggest that centriole elimination results from PCM loss. Upon mitotic entry, Polo-like kinase 1 (called PLK1 in most species, Polo in Drosophila) is known to be a major regulator of $\operatorname{PCM}$ recruitment to the centriole $(25,26)$. Polo and its orthologues directly phosphorylate several core scaffold PCM proteins in different organisms, including Pericentrin (PLP), SPD2 and CNN, contributing for $\gamma$-tubulin accumulation on centrosomes, PCM assembly and expansion (16, 21, 27-31). PLK1 was shown recently to also play a role in PCM maintenance (21, 32).

In Drosophila oogenesis Polo is required at early stages to restrict meiosis to the oocyte (33), and later on in stage 14 to trigger nuclear envelope breakdown (34). Interestingly, as Polo was shown recently to be transcriptionally down-regulated in the oocyte in between those stages (35), it is possible that Polo is absent from centrosomes, leading to PCM disappearance and centriole loss. We therefore examined Polo's subcellular localization in those stages. We observed that while $89 \%$ of early stage oocytes (stages 2 to 6 ) showed the presence of Polo at the centrioles (Fig. 2A,B), its centriolar localization decreased dramatically in mid and late stages of oogenesis (Fig. 2A,B), which coincides with our observations on PCM loss (Fig. 1I). Importantly, most centrioles disappear from the oocyte between stage 10 and stage 12/13 (see Fig. 1J), when Polo is absent from the MTOC. These observations suggest that Polo loss from the oocyte's centrosome could be a critical event in triggering loss of the PCM, followed by centriole elimination.

Down-regulating Polo accelerates centriole loss

We then tested whether Polo could have a role in centriole maintenance. Polo mutants have a strong loss of function phenotype early in oogenesis (33), precluding an analysis of 
the effect of its loss of function in centriole maintenance. We therefore used RNAi in order to down-regulate Polo only after oocyte determination when Polo is naturally still present. RNAi depletion led to loss of Polo protein (fig. S3A) and to female sterility, as reported previously for mutants of Polo (27). We analyzed centriole maintenance by investigating the levels of two centriole markers, ANA1-tdtomato and PACT-GFP (the centriole targeting domain of pericentrin $(14,31,36,37))$. We focused on stage 10 where normally centrioles are still present (see Fig 1J). We observed that the levels of both markers were significantly reduced upon Polo down-regulation in contrast to a mCherry control, strongly suggesting the presence of less centrioles and thus acceleration of centriole loss (Fig. 2C, D). We observed very similar results in tissue culture cells upon down-regulation of polo (fig. S4). As a read-out we used a centriole marker, BLD10, a centriole and PCM marker, SAS4, and a PCM marker, D-PLP (fig. S4). Polo depletion alone had an effect on PCM depletion, similar to its effect on centriole loss (fig. S4A,B). Furthermore, co-depletion of Polo and 'All PCM' led to a similar phenotype as compared to the one obtained with depletion of 'All PCM' alone, suggesting that Polo and PCM work on the same pathway to maintain centrioles (fig. S4A,B).

\section{Ectopic tethering of Polo to centrioles prevents PCM loss}

To further test the role of Polo at the centrosome for PCM and centriole maintenance, we asked whether overexpression of this kinase (Polo-Myc) throughout oogenesis (starting at stages 3/4), could overcome centriole loss. However, despite an increase in total Polo levels (fig. S3B), Polo did not localize to most oocyte centrioles in late stages (fig. S3D) and induced only a modest increase in the percentage of late oocytes showing $\boldsymbol{Y}$-tubulin on centrioles (fig. S3C). As Polo is a very dynamic protein, localizing to different subcellular structures at different cell cycle stages (26), we reasoned that overexpression of Polo might not be sufficient to force its concentration to the centrioles and thus to retain the PCM there.

We took advantage of an approach to force Polo to the oocyte's centrioles. Several molecules, including the orthologue of Polo, PLK1, have been artificially tethered to the centriole by fusion to pericentrin or its centriole-targeting domain, PACT $(14,31$, 36, 37). We therefore targeted Polo to the centriole by fusing GFP-Polo to PACT. This strategy worked in Drosophila tissue culture cells where expression of GFP-Polo-PACT led 
to PCM accumulation, even in interphasic centrioles. Moreover it did not elicit changes in mitotic progression or centrosome number (not shown). We therefore induced GFP-PoloPACT expression in oogenesis. GFP-Polo-PACT and GFP-PACT (control) always localized to the oocyte's centrioles from stages 2 to 12 (GFP signal always co-localized with ANA1tdTomato, Fig. 3A). We then asked whether the constant presence of Polo at the centrioles could prevent PCM loss, from hereinafter also called loss of PCM maintenance. We used $\gamma$-tubulin as a read-out, as this component is downstream of the other PCM constituents (Fig. 1G,I). Additionally, we detailed the characterization of mid and very late (less abundant) stages of oogenesis, when centrosome components start to disappear. We observed a remarkable effect in the presence and levels of $\gamma$-tubulin at the ANA1 and GFP-Polo-PACT co-localizing centrioles: in very late and less abundant stages (12 to 13), where we rarely observed GFP-PACT oocytes (control) containing centrioles with $\mathrm{Y}^{-}$ tubulin, $90 \%$ of the GFP-Polo-PACT oocytes showed centrioles with $\gamma$-tubulin (Fig. 3A,B). Moreover, centriolar $\gamma$-tubulin levels were increased in oocytes expressing GFP-Polo-PACT (Fig. 3A,C). We also observed that SPD2 was retained until later than in controls (not shown). We conclude that tethering Polo to the oocytes centrioles prevents PCM loss in mid and late oogenesis.

\section{Polo-dependent PCM maintenance prevents centriole elimination}

We then asked whether maintaining centrioles coated by PCM would prevent their normal elimination. We quantified the levels and presence of the core centriolar protein ANA1 in mid and late oogenesis as before (Fig. 3A,D,E). Upon expression of GFP-PoloPACT, ANA1 levels in mid stages (7/8) were very similar to the control (GFP-PACT, Fig. 3D; roughly equivalent to 50 early stage centrioles, Fig. 1J). Strikingly, in late stages (12 to 13), while $68 \%$ of control oocytes had few remaining centrioles (considering ANA1 levels) (Fig. 3D), all GFP-Polo-PACT expressing oocytes retained centrioles (Fig. 3E) and had similar levels of ANA1 signal distributed in different clusters (Fig. 3D). Importantly, the fact that ANA1 total signal in the oocyte does not change throughout oogenesis when GFP-PoloPACT is expressed (Fig. 3D) strongly suggests that there is neither significant centriole loss, in contrast to what is observed in the control, nor extra centriole biogenesis (both canonical or de novo), at any time point along oogenesis. Moreover, we also observed that centrioles were maintained in Polo-PACT expressing eggs, even after PLK4, a critical 
player in centriole biogenesis, was down-regulated in ovaries through RNAi (not shown), strongly suggesting there is no additional centriole formation throughout oogenesis.

Centrioles have an unequivocal cylindrical structure that due to their very small size ( $250 \mathrm{~nm}$ diameter across, approximately $400 \mathrm{~nm}$ long), their walls cannot be resolved by conventional light microscopy. They have been traditionally resolved by electron microscopy, or more recently by super-resolution microscopy. We developed a protocol to use super-resolution microscopy (Structured Illumination Microscopy - SIM) in ovaries to validate the presence of normal centrioles by resolving the walls of the cylinder, a unique structure in the cell. We focused on stage 12, where normally most centrioles have been eliminated (Fig. 1J). Centriole barrels were clearly identified at stage 12 both in the control (GFP-PACT) and in GFP-Polo-PACT (Fig. 4A).

We then asked whether expression of GFP-Polo-PACT would also ensure centriole maintenance after meiotic nuclear envelope breakdown. Consistent with our initial characterization (Fig. 1F), in control oocytes (GFP-PACT) we were unable to identify centrioles at the spindle poles of meiosis I (stage 14) (Fig. 3F,G, left panels). Strikingly, in GFP-Polo-PACT expressing oocytes we always observed centrioles (Fig. 3F,G, right panels). Centrioles were mostly located at the anterior end of the immature egg, in the vicinity of the DNA and generally more scattered than in previous stages, forming on average 20 centriole clusters (Fig. 3F,G; fig. S5A,B). In some eggs, centrioles were no longer clustered and their number could be resolved with a maximum of 56 centrioles being observed in one egg (not shown). Moreover, we could observe the centriole barrel in this stage in GFP-Polo-PACT eggs in super-resolution micrographs (Fig. 4A, right panel), but no centrioles were observed in this stage in GFP-PACT controls (not shown), as expected from conventional light microscopy experiments (Fig. 1F and $3 F, G$ ). Those centrioles, in contrast to the remnant centrioles encountered in control eggs (fig. S1E), were able to nucleate MTs (fig. S5B). These results strongly suggest that the majority of the centrioles were maintained as fully competent MTOCs.

Polo and its orthologues are known to have several functions mediated by catalysis. However, it has been speculated that some members of this family, in particular PLK5 can have non-catalytic functions $(38,39)$. We asked whether Polo activity is critical for its function in centriole maintenance in both tissue culture cells and eggs. We generated a catalytically dead GFP-Polo-PACT (GFP-Polo-KD-PACT) as described before 
(37). Expression of this construct at equal levels to GFP-Polo-PACT in ovaries was not able to fully rescue the centriole loss that normally occurs in stage $12 / 13$, enforcing the importance of Polo catalytic activity in centriole maintenance (fig. S6). Given that there was less centriole loss in the kinase dead as compared to controls, it is possible that Polo also has some non-catalytic function in centriole maintenance. Similar results were obtained in tissue culture cells (fig. S7). Therefore, we can conclude, that centriole elimination requires Polo loss from the centrioles.

\section{Polo-dependent centriole maintenance leads to abnormal meiosis and aborted embryonic development}

We then focused on the consequences of retaining centrioles for meiosis and embryo development. In GFP-Polo-PACT expressing eggs, while few meiosis looked normal upon centriole maintenance, centrioles often seemed to interact with the spindle, leading to abnormal meiosis, in many cases with scattered DNA (right panel in Fig. 4B and fig. S5A). Therefore, centriole elimination at late oogenesis requires Polo loss and consequent PCM loss. Moreover, non-eliminated centrioles can interfere with meiotic spindle assembly.

We then asked what would be the consequences for reproduction of retaining centrioles in the egg. Unfertilized eggs did not show any obvious morphological defects and the dorsal appendages of the chorion (specialized structures in the $D$. melanogaster egg that ensure the breathing of the embryo), a hallmark of proper egg development, were well formed (not shown), suggesting that ectopic centrosome presence during oogenesis did not induce major polarity defects. It is known that injection of centrioles in Xenopus eggs induces parthenogenic development (7). However, we did not observe parthenogenic offspring from unfertilized Drosophila eggs retaining centrosomes (GFPPolo-PACT; not shown). Fertilized eggs having both a maternal (GFP-Polo-PACT expressing eggs) and a paternal centrosome were laid in similar amounts when comparing with the control (Fig. 4C). However, eggs expressing GFP-Polo-PACT showed a very low egg hatching rate of $1 \%$, when compared to $75 \%$ in GFP-PACT control (Fig. 4D), and to $54 \%$ in Polo-Myc overexpression, where Polo was not tethered to centrioles (fig. S5D). While eggs were fertilized (fig. S8A), we observed that embryogenesis was blocked very early in development (Fig. 4B). The majority of embryos from GFP-Polo-PACT-expressing mothers arrested in the first mitotic divisions (Fig. 4B; fig. S5E), often with multiple centrosomes at 
each pole and scattered DNA associated with centrosomes (Fig. 4B), probably resulting from abnormal chromosome segregation. GFP-Polo-PACT expression per se, at the levels observed, is unlikely to have major detrimental effects in embryonic development, given the presence of normal escaper embryos (fig. S8B). These results lead us to conclude that maternal centrosome maintenance is detrimental for female meiosis and early embryonic development, having a negative impact on sexual reproduction.

\section{DISCUSSION}

Asymmetric centriole inheritance is thought to be essential to sexual reproduction. How maternal centrioles are eliminated and the importance of this phenomenon for oogenesis progression, meiotic division and embryogenesis, has been a matter of extensive debate. Here we show for the first time that maternal Drosophila centriole elimination results from the shutdown of a novel centriole maintenance program relying on the presence of Polo kinase at the centrosome and consequent PCM retention (Fig. 4E). By artificially maintaining this program active by tethering Polo to centrioles, we retained maternal centrosomes throughout all oogenesis. Surprisingly, eggs with active centrosomes are well patterned and laid. However, the abnormal presence of centrosomes leads to defective meiosis, abnormal mitosis after fertilization and aborted early embryonic development (Fig. 4E).

Our findings are likely to extend to the female germline of other animals, where centrioles are also eliminated during prophase I arrest. Accordingly, in C. elegans oocytes the PCM is also lost before centrioles are eliminated (9), and in Xenopus oocytes PIX1 (Polo orthologue) is also less expressed in early oogenesis $(40,41)$. Echinoderms are an exception to centriole elimination in early prophase I arrest, as most centrioles are eliminated through extrusion within the polar bodies during both meiotic divisions, with a last centriole being eliminated after meiosis exit (8). Remarkably, echinoderm centrioles are surrounded by PCM ( $\gamma$-tubulin and pericentrin) in all stages of oogenesis (42), which may protect them, further supporting the generality of a PCM-dependent centriole maintenance mechanism. It is also known that Polo substrate phosphorylation is important, both for their localization and to reinforce Polo's own localization at the centrosome, in a positive feedback loop (26). Recently, it was shown that both Polo and 
at least one of its PCM substrates, SPD2, are not transcribed at the beginning of late stages of Drosophila oogenesis (stages 9 and 10) (35). We propose that concomitant loss of expression of Polo and its PCM substrates leads to complete PCM loss from the oocyte MTOC and subsequent centriole loss. Moreover, physiological inhibitors of Polo (such as matrimony) (34) may contribute to further down-regulate its localization and activity at the centriole. Future work will focus on understanding, which Polo substrates and binding proteins are important in this function.

To our knowledge, animal sexual reproduction is always associated with asymmetric centriole inheritance due to maternal centriole loss. Here, we tested the consequences of counteracting this process for meiosis and early embryonic development. We observed that oogenesis is robust to the presence of active centrioles (no major patterning defects). However, maternal centrioles interfere with meiotic and mitotic divisions in the embryo, leading to female sterility. Moreover, the presence of maternal centrioles is not sufficient to support parthenogenesis in Drosophila melanogaster. In parthenogenic species, centrioles are still eliminated in oogenesis and appear de novo only after anaphase I (43). It is thus possible that the meiotic defects observed caused by the presence of maternal centrioles, or other unrelated requirements, preclude the induction of parthenogenesis.

Our study revealed that the widely accepted centriole stability is not an intrinsic property of those structures. Instead, centrioles are maintained by a novel program, dependent on a new interphase role by the major mitotic kinase Polo and by the MT nucleating matrix that surrounds the centriole, the PCM. We propose this is a general feature of centrioles, as we observed that either Polo or PCM removal lead to centriole disappearance in Drosophila interphasic somatic cells arrested for a long time (fig. S2 and S4). Further studies are needed to address the novel role of PCM and Polo in centriole maintenance and how that interplays with the role of centriole stabilizing structural features (44-47). Finally, evidence of PCM's presence or loss from centrosomes in different tissues supports the idea that regulation of a centriole maintenance program is present and critical in many other cell types, such as cells that lose centrioles or their activity upon differentiation or disease (e.g. muscle, virus infection, cells with multiple centrosomes in cancer) and in cells that keep centrioles for a long time (e.g. cycling cells 
where centrioles go through many cell cycles and long-lived differentiated ciliated cells) $(6,48-52)$.

\section{Acknowledgements}

We are thankful to Meng-Fu Bryan Tsou, Rui Martinho, Raquel Oliveira, Alex Dammermann, Trudi Schupbach, Peter Lenart, Joana Pinto, Jens Januschke, Vincent Archambault, Pedro Prudêncio, Maria Francia, Mariana Lince-Faria, José Pereira-Leal and Elio Sucena for critical reading of the manuscript and all the members of MB-D laboratory for discussions. We would also like to thank Hiro Ohkura for sharing protocols and Adelaide Carpenter and Jeroen Dobbelaere for discussions and sharing unpublished data. We thank Tomer Avidor Reiss, Jordan Raff, Daniel St Johnston and Vincent Archambault for sharing tools. Both ARM and SJ are funded by postdoctoral fellowships (SFRH/BPD/79680/2011 and SFRH/BPD/87479/2012, respectively). IB is funded by a FCT doctoral fellowship. M.B-D. and the Cell Cycle Regulation laboratory (CCR) are supported by an EMBO installation grant, an ERC grant ERC-2010-StG-261344 and grants from the Fundação para a Ciência e a Tecnologia (FCT - Portugal): Ciencia 2007 program; FCTinvestigator, PTDC/SAU-BD/105616/2008. 


\section{REFERENCES}

1. M. Sato, K. Sato, Dynamic regulation of autophagy and endocytosis for cell remodeling during early development. Traffic 14, 479-486 (2013).

2. D. A. Brito, S. M. Gouveia, M. Bettencourt-Dias, Deconstructing the centriole: structure and number control. Curr Opin Cell Biol 24, 4-13.

3. M. Delattre, P. Gonczy, The arithmetic of centrosome biogenesis. J Cell Sci 117, 1619-1630 (2004).

4. G. Manandhar, H. Schatten, P. Sutovsky, Centrosome reduction during gametogenesis and its significance. Biol Reprod 72, 2-13 (2005).

5. A. Rodrigues-Martins, M. Riparbelli, G. Callaini, D. M. Glover, M. Bettencourt-Dias, From centriole biogenesis to cellular function: centrioles are essential for cell division at critical developmental stages. Cell Cycle 7, 11-16 (2008).

6. I. Cunha-Ferreira, I. Bento, M. Bettencourt-Dias, From zero to many: control of centriole number in development and disease. Traffic 10, 482-498 (2009).

7. F. Tournier, E. Karsenti, M. Bornens, Parthenogenesis in Xenopus eggs injected with centrosomes from synchronized human lymphoid cells. Dev Biol 136, 321-329 (1989).

8. J. Borrego-Pinto et al., Distinct mechanism eliminate mother and daughter centrioles in meiosis of starfish oocytes. J Cell Biol, (2016, In Press).

9. T. Mikeladze-Dvali et al., Analysis of centriole elimination during C. elegans oogenesis. Development 139, 1670-1679 (2012).

10. A. F. Huettner, M. Rabinowitz, Demonstration of the Central Body in the Living Cell. Science 78, 367-368 (1933).

11. A. P. Mahowald, J. M. Strassheim, Intercellular migration of centrioles in the germarium of Drosophila melanogaster. An electron microscopic study. J Cell Biol 45, 306-320 (1970).

12. J. Januschke et al., The centrosome-nucleus complex and microtubule organization in the Drosophila oocyte. Development 133, 129-139 (2006).

13. S. Blachon et al., Drosophila asterless and vertebrate Cep152 Are orthologs essential for centriole duplication. Genetics 180, 2081-2094 (2008).

14. M. Martinez-Campos, R. Basto, J. Baker, M. Kernan, J. W. Raff, The Drosophila pericentrinlike protein is essential for cilia/flagella function, but appears to be dispensable for mitosis. J Cell Biol 165, 673-683 (2004).

15. E. P. Lucas, J. W. Raff, Maintaining the proper connection between the centrioles and the pericentriolar matrix requires Drosophila centrosomin. J Cell Biol 178, 725-732 (2007).

16. P. T. Conduit et al., The centrosome-specific phosphorylation of Cnn by Polo/Plk1 drives Cnn scaffold assembly and centrosome maturation. Dev Cell 28, 659-669 (2014).

17. C. I. Dix, J. W. Raff, Drosophila Spd-2 recruits PCM to the sperm centriole, but is dispensable for centriole duplication. Curr Biol 17, 1759-1764 (2007).

18. M. G. Giansanti, E. Bucciarelli, S. Bonaccorsi, M. Gatti, Drosophila SPD-2 is an essential centriole component required for PCM recruitment and astral-microtubule nucleation. Curr Biol 18, 303-309 (2008).

19. J. Gopalakrishnan et al., Sas-4 provides a scaffold for cytoplasmic complexes and tethers them in a centrosome. Nat Commun 2, 359 (2011).

20. N. S. Dzhindzhev et al., Asterless is a scaffold for the onset of centriole assembly. Nature 467, 714-718.

21. J. B. Woodruff, O. Wueseke, A. A. Hyman, Pericentriolar material structure and dynamics. Philos Trans R Soc Lond B Biol Sci 369, (2014).

22. J. J. Moser, M. J. Fritzler, Y. Ou, J. B. Rattner, The PCM-basal body/primary cilium coalition. Semin Cell Dev Biol 21, 148-155.

23. Y. Shang, B. Li, M. A. Gorovsky, Tetrahymena thermophila contains a conventional gamma-tubulin that is differentially required for the maintenance of different microtubule-organizing centers. J Cell Biol 158, 1195-1206 (2002). 
24. J. Fu, D. M. Glover, Structured illumination of the interface between centriole and pericentriolar material. Open Biol 2, 120104 (2012).

25. W. J. Wang, R. K. Soni, K. Uryu, M. F. Tsou, The conversion of centrioles to centrosomes: essential coupling of duplication with segregation. J Cell Biol 193, 727-739.

26. S. Zitouni, C. Nabais, S. C. Jana, A. Guerrero, M. Bettencourt-Dias, Polo-like kinases: structural variations lead to multiple functions. Nat Rev Mol Cell Biol 15, 433-452.

27. C. E. Sunkel, D. M. Glover, polo, a mitotic mutant of Drosophila displaying abnormal spindle poles. J Cell Sci 89 ( Pt 1), 25-38 (1988).

28. H. A. Lane, E. A. Nigg, Antibody microinjection reveals an essential role for human pololike kinase 1 (Plk1) in the functional maturation of mitotic centrosomes. $J$ Cell Biol 135, 1701-1713 (1996).

29. L. Haren, T. Stearns, J. Luders, Plk1-dependent recruitment of gamma-tubulin complexes to mitotic centrosomes involves multiple PCM components. PLoS One 4, e5976 (2009).

30. J. Dobbelaere et al., A genome-wide RNAi screen to dissect centriole duplication and centrosome maturation in Drosophila. PLoS Biol 6, e224 (2008).

31. K. Lee, K. Rhee, PLK1 phosphorylation of pericentrin initiates centrosome maturation at the onset of mitosis. J Cell Biol 195, 1093-1101 (2011).

32. R. Mahen, A. D. Jeyasekharan, N. P. Barry, A. R. Venkitaraman, Continuous polo-like kinase 1 activity regulates diffusion to maintain centrosome self-organization during mitosis. Proc Natl Acad Sci U S A 108, 9310-9315 (2011).

33. V. Mirouse, E. Formstecher, J. L. Couderc, Interaction between Polo and BicD proteins links oocyte determination and meiosis control in Drosophila. Development 133, 40054013 (2006).

34. Y. Xiang et al., The inhibition of polo kinase by matrimony maintains $\mathrm{G} 2$ arrest in the meiotic cell cycle. PLoS Biol 5, e323 (2007).

35. H. Jambor et al., Systematic imaging reveals features and changing localization of mRNAs in Drosophila development. Elife 4, (2015).

36. A. K. Gillingham, S. Munro, The PACT domain, a conserved centrosomal targeting motif in the coiled-coil proteins AKAP450 and pericentrin. EMBO Rep 1, 524-529 (2000).

37. K. Kishi, M. A. van Vugt, K. Okamoto, Y. Hayashi, M. B. Yaffe, Functional dynamics of Pololike kinase 1 at the centrosome. Mol Cell Biol 29, 3134-3150 (2009).

38. V. Archambault, G. Lepine, D. Kachaner, Understanding the Polo Kinase machine. Oncogene 34, 4799-4807 (2015).

39. G. de Carcer, G. Manning, M. Malumbres, From Plk1 to Plk5: functional evolution of pololike kinases. Cell Cycle 10, 2255-2262 (2011).

40. A. Karaiskou et al., Polo-like kinase confers MPF autoamplification competence to growing Xenopus oocytes. Development 131, 1543-1552 (2004).

41. D. L. Gard, D. Affleck, B. M. Error, Microtubule organization, acetylation, and nucleation in Xenopus laevis oocytes: II. A developmental transition in microtubule organization during early diplotene. Dev Biol 168, 189-201 (1995).

42. A. L. Egana, J. A. Boyle, S. G. Ernst, Strongylocentrotus drobachiensis oocytes maintain a microtubule organizing center throughout oogenesis: implications for the establishment of egg polarity in sea urchins. Mol Reprod Dev 74, 76-87 (2007).

43. M. G. Riparbelli, G. Callaini, Drosophila parthenogenesis: a model for de novo centrosome assembly. Dev Biol 260, 298-313 (2003).

44. P. Guichard et al., Native architecture of the centriole proximal region reveals features underlying its 9-fold radial symmetry. Curr Biol 23, 1620-1628 (2013).

45. D. Izquierdo, W. J. Wang, K. Uryu, M. F. Tsou, Stabilization of cartwheel-less centrioles for duplication requires CEP295-mediated centriole-to-centrosome conversion. Cell Rep 8, 957-965 (2014). 
46. V. Mennella et al., Subdiffraction-resolution fluorescence microscopy reveals a domain of the centrosome critical for pericentriolar material organization. Nat Cell Biol 14, 11591168 (2012).

47. S. Li, J. J. Fernandez, W. F. Marshall, D. A. Agard, Three-dimensional structure of basal body triplet revealed by electron cryo-tomography. EMBO J 31, 552-562 (2012).

48. R. H. Warren, Microtubular organization in elongating myogenic cells. J Cell Biol 63, 550566 (1974).

49. R. J. Przybylski, Occurrence of centrioles during skeletal and cardiac myogenesis. J Cell Biol 49, 214-221 (1971).

50. A. M. Tassin, B. Maro, M. Bornens, Fate of microtubule-organizing centers during myogenesis in vitro. J Cell Biol 100, 35-46 (1985).

51. J. A. Connolly, B. W. Kiosses, V. I. Kalnins, Centrioles are lost as embryonic myoblasts fuse into myotubes in vitro. Eur J Cell Biol 39, 341-345 (1986).

52. V. Brodu, A. D. Baffet, P. M. Le Droguen, J. Casanova, A. Guichet, A developmentally regulated two-step process generates a noncentrosomal microtubule network in Drosophila tracheal cells. Dev Cell 18, 790-801 (2010). 


\section{FIGURES}

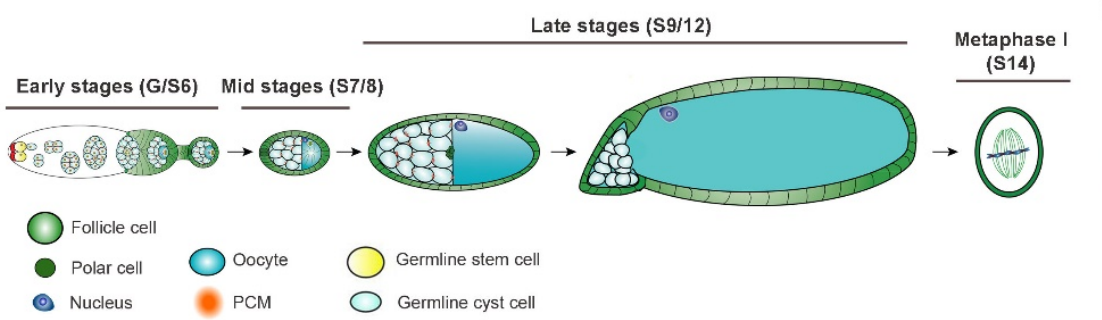

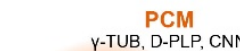

-TUB, D-PLP, CNN

ASL, SAS4, SPD2

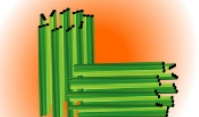

Centriole

ANA1, SAS6, BLD10

c
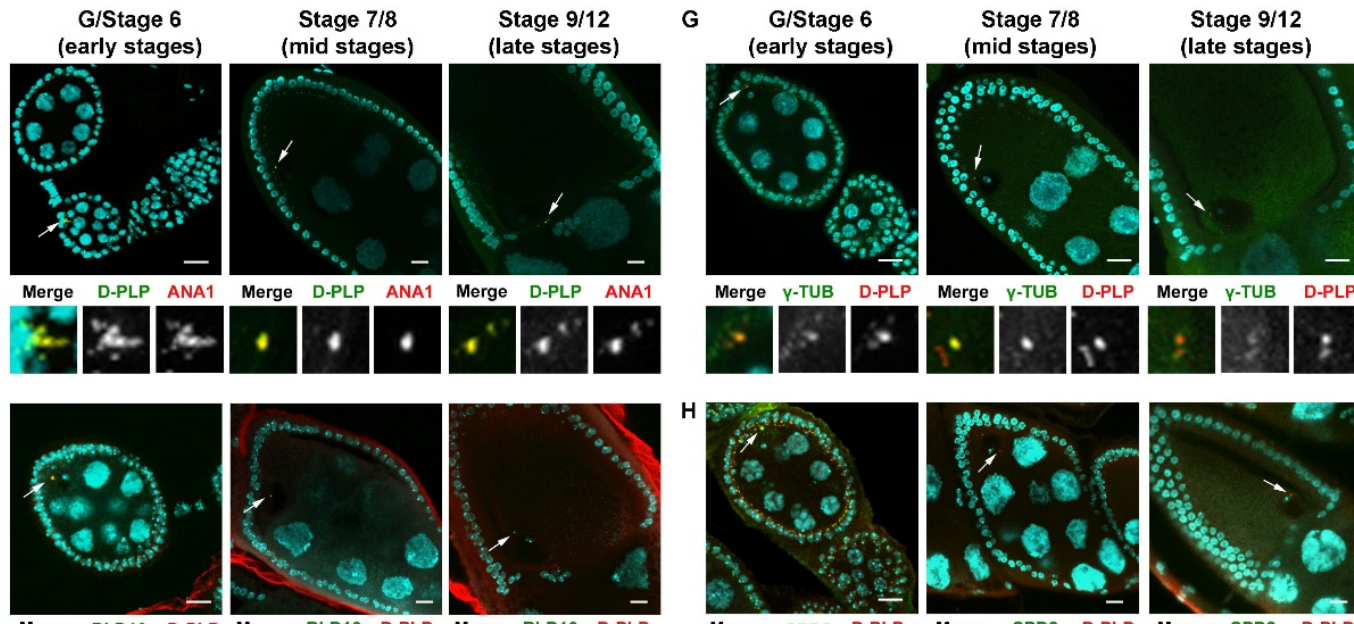

Merge BLD10 D-PLP Merge BLD10 D-PLP Merge BLD10 D-PLP
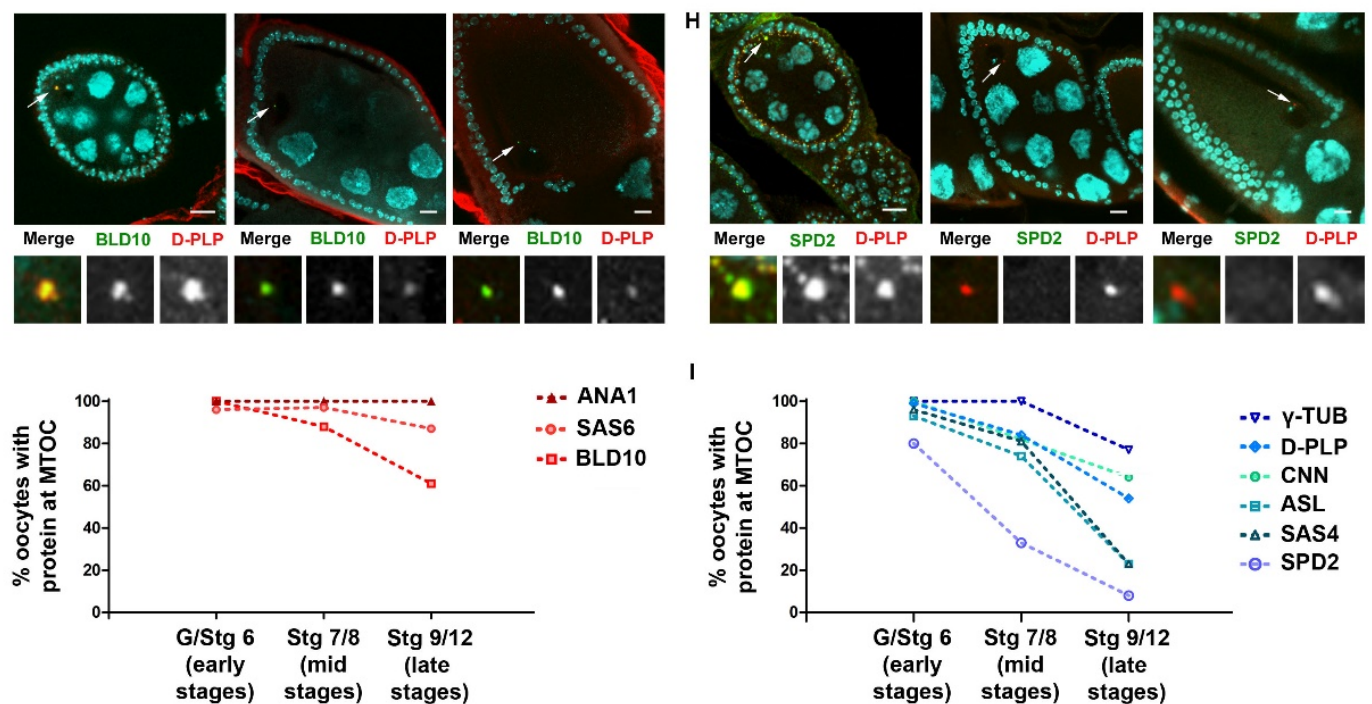

$\mathbf{F}$

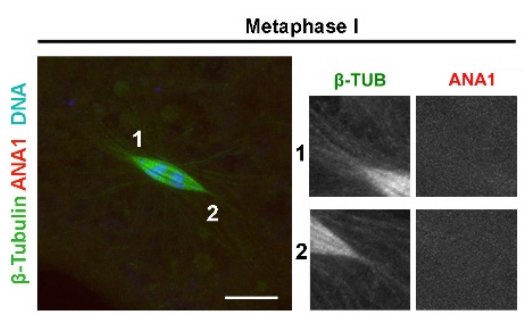
-०- SAS6 - BLD10

$J$

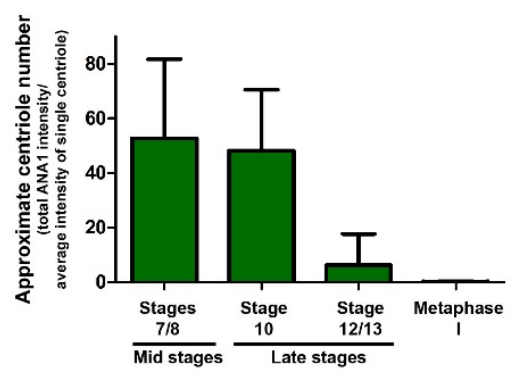

Fig. 1. Centrosomes progressively disassemble at the end of oogenesis: PCM first, centrioles last. (A) Drosophila melanogaster oogenesis. Oogenesis begins with the asymmetric division of the stem cell, producing a cystoblast that divides four times to create a cyst of 16 interconnected cells, one of which becomes the oocyte (blue) while the others become nurse cells (grey). Early in oogenesis the centrioles of the nurse cells migrate to the oocyte (see text), where a complex microtubule (MT) rearrangement takes place (Germarium (G) to Stage 6 (S6)). Centrioles were observed as late as stages 8 and 9 \{Januschke, $2006 \# 56$ \}. The presence of centrioles in pre 
nuclear envelope breakdown (NEBD) stages (S13/14) remains uncharacterized, while meiosis (I and II) are known to be acentriolar. Images adapted from \{Cunha-Ferreira, 2009 \#13\}. (B) Centrosome structure and components. The centrosome is composed by two centrioles, which are made of 9 triplets of MTs (green), and by the PCM (orange), a matrix of proteins whose known function is to nucleate and anchor MTs. (C and D) ANA1 (C) and BLD10 (D), core centriole components, are detected close to the oocyte's nucleus from early to late stages (from $\mathbf{G}$ to S12). Note the increase in the size of the oocyte, the large nuclei of the nurse cells and the follicle cells surrounding the egg. Enlargements of the indicated areas (arrows) are shown (5.3x and 7.5x magnification in early stages and mid/late stages, respectively, so that the images are set to comparable scales). Note the gradual decrease of the intensity and size of centriole-containing structures (images were acquired with same exposure). Scale bars, $10 \mu \mathrm{m}$. (E) Quantification of oocytes showing ANA1, SAS6 or BLD10 as discrete foci in the different stages. More than 30 independent oocytes were analyzed per stage, per protein. Due to their long duration, most oocytes counted in late stages were either in stage 9 or 10. Dashed lines are included to help infer general trends. (F) Female meiosis I. Note that meiosis I is acentriolar (no centrioles at the poles). Enlargements (1.5x magnification) of the indicated areas are shown. (See also fig. S1 for SAS6 staining.) (G and H) Localization of $\gamma$-tubulin ( $\gamma$-TUB) (G) and SPD2 (H), PCM markers, in the oocyte. While $\gamma$-TUB is very stable, SPD2 is absent from the majority of oocytes from mid and late stages. Note the gradual decrease of the intensity and size of the PCM. Enlargements of the indicated areas (arrows) are shown (5.3x and 7.5x magnification in early stages and mid/late stages, respectively, so that the images are set to comparable scales). Scale bars, $10 \mu \mathrm{m}$. (I) Quantification of the oocytes positive for PCM components: $p$-TUB, D-PLP, CNN, ASL, SAS4 and SPD2. Note that the presence of PCM proteins decreases in mid stage oocytes. More than 30 independent oocytes were analyzed per stage, per protein. Due to their long duration, most oocytes counted in late stages were either in stage 9 or 10. Dashed lines are included to help infer general trends. (See also fig. S1 for SAS4, ASL and CNN staining.) (J) Estimate of total centriole number in the oocyte at different stages. Up until stage 10 centrioles are found in very tight clusters, being impossible to discriminate their number with light microscopy. To estimate centriole numbers we divided total intensity of ANA1 at each stage by the average intensity of single centrioles encountered in early stages in nurse cells. GFP-PACT was used as a centriole marker, ensuring co-localization (16). 
A

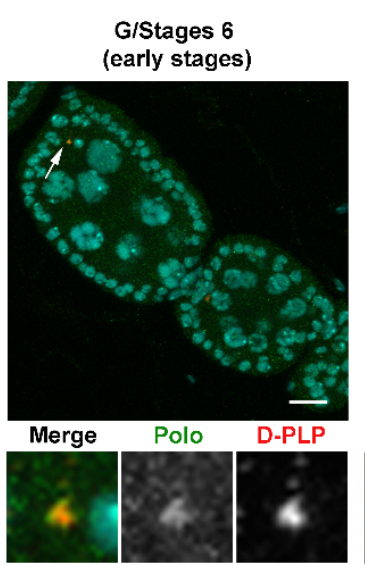

c

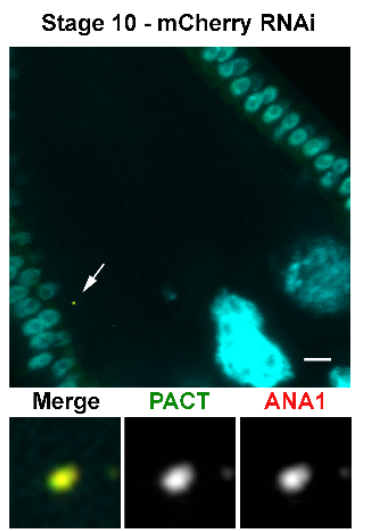

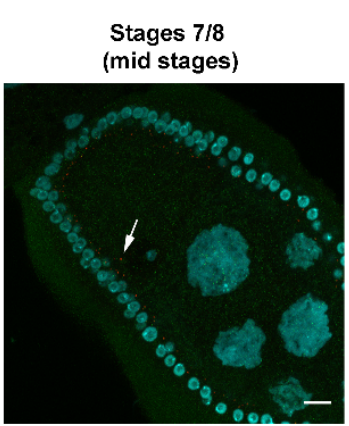

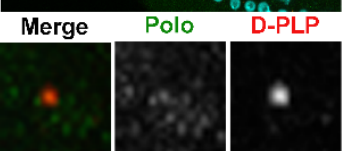

Stage 10 - Polo RNAi

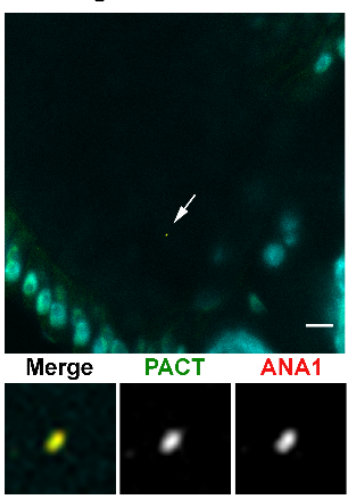

Stage $9 / 12$ (late stages)

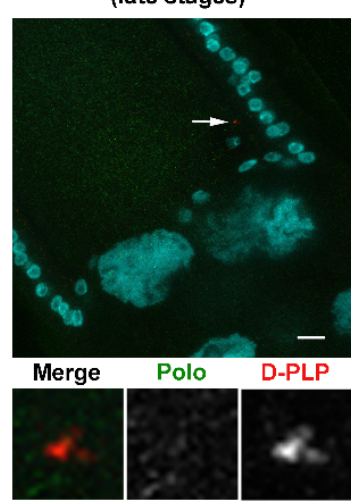

D

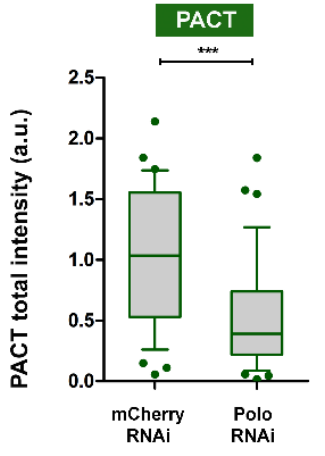

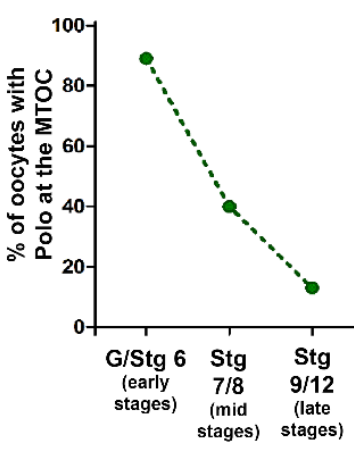

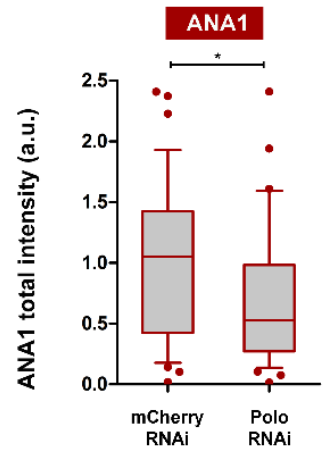

Fig. 2. Centrosomal Polo is naturally down-regulated in mid and late stages of oogenesis; downregulation of Polo accelerates centriole loss. (A) Localization of endogenous Polo. Oocytes were immunostained for Polo and D-PLP (clustered centrosomes, i.e. oocyte MTOC). Enlargements of the indicated areas (arrows) are shown (5.8x and 8.5x magnification in early stages and $\mathrm{mid} /$ late stages, respectively, so that the images are set to comparable scales). All images were acquired with same exposure. Scale bars, $10 \mu \mathrm{m}$. (B) Quantification of oocytes positive for Polo at the oocyte MTOC. Note that the majority of late stage oocytes do not have Polo at the MTOC. Dashed lines are included to help infer general trend. (C) Depletion of Polo by RNAi. mCherry-RNAi (control) and Polo-RNAi were expressed in the germline using a driver that only expresses after stage $3 / 4$, i.e. after oocyte specification. Expression of both GFP-PACT (under poliubiquitin promoter; PACT is the centriolar targeting domain of PLP (36)) and ANA1-tdTomato (under endogenous promoter) were used as robust centriolar markers. Enlargements (5x magnification) of the indicated areas (arrows) are shown. All images were acquired with same exposure. We investigated the presence of centrioles at stage 10, before they normally disappear (see figure 1J). Note the smaller size of MTOCs after Polo RNAi in comparison to the control (mCherry RNAi), seen both with the PACT and Ana1 centriole markers. Scale bars, $10 \mu \mathrm{m}$. Western blot showing Polo depletion is shown in fig S3A. (D) Quantification of total co-localized GFP-PACT and ANA1tdTomato levels as proxy for centriole numbers in stages 10 . A minimum of 30 oocytes were analyzed per RNAi condition. The statistical difference between samples was evaluated with a ttest $\left(* * *, p<0.001 ;{ }^{*}, p<0.05\right)$. 

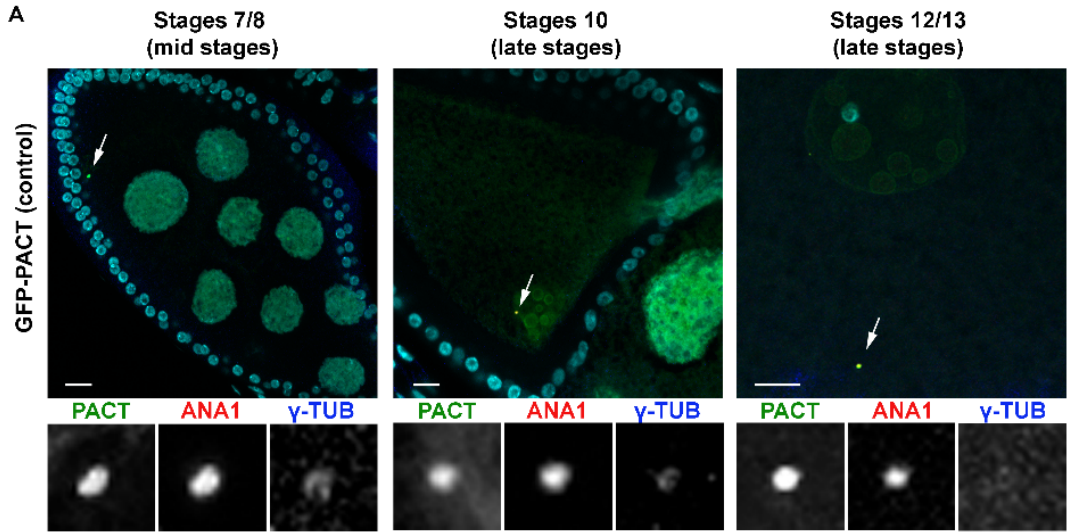

B
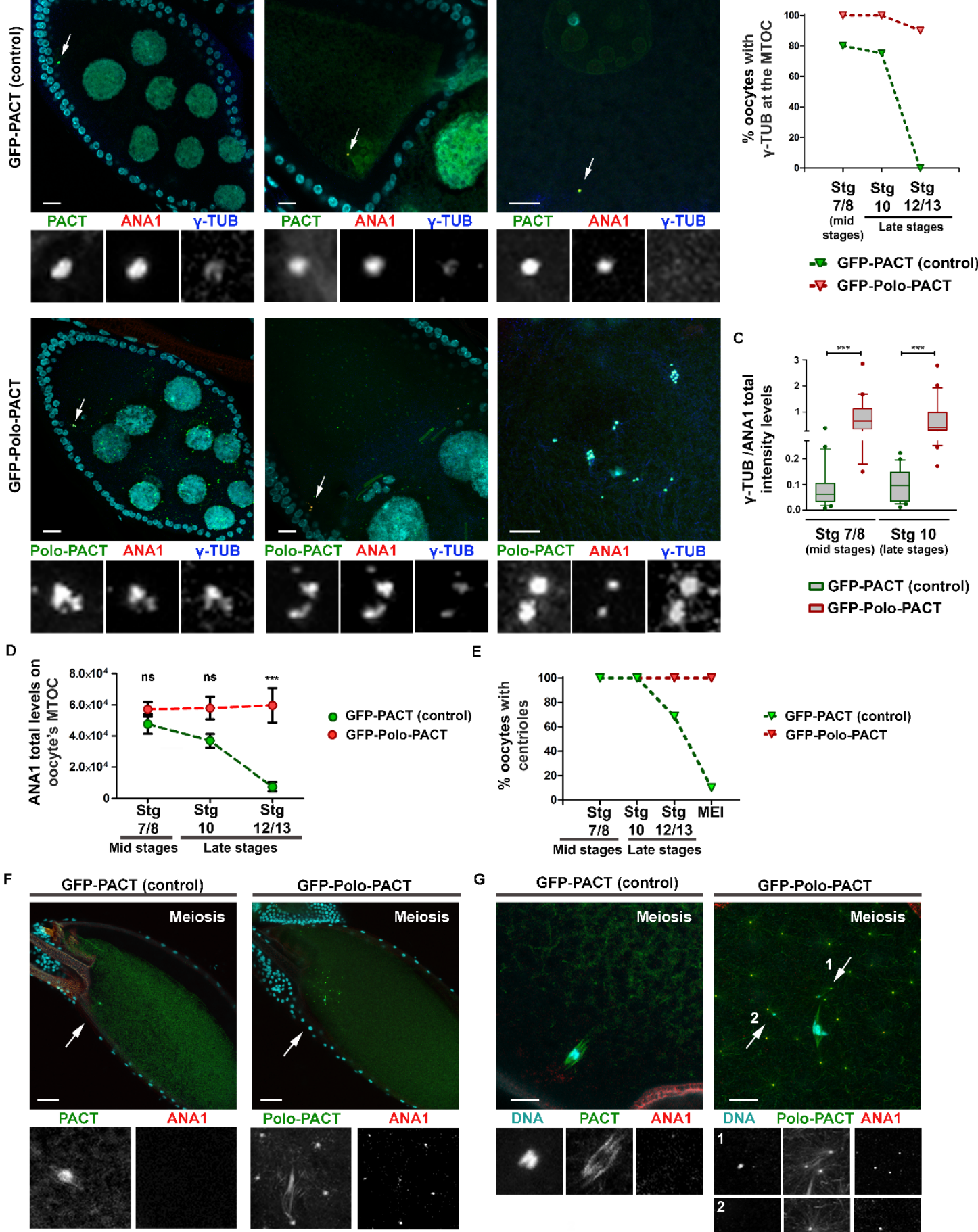

G GFP-PACT (control)
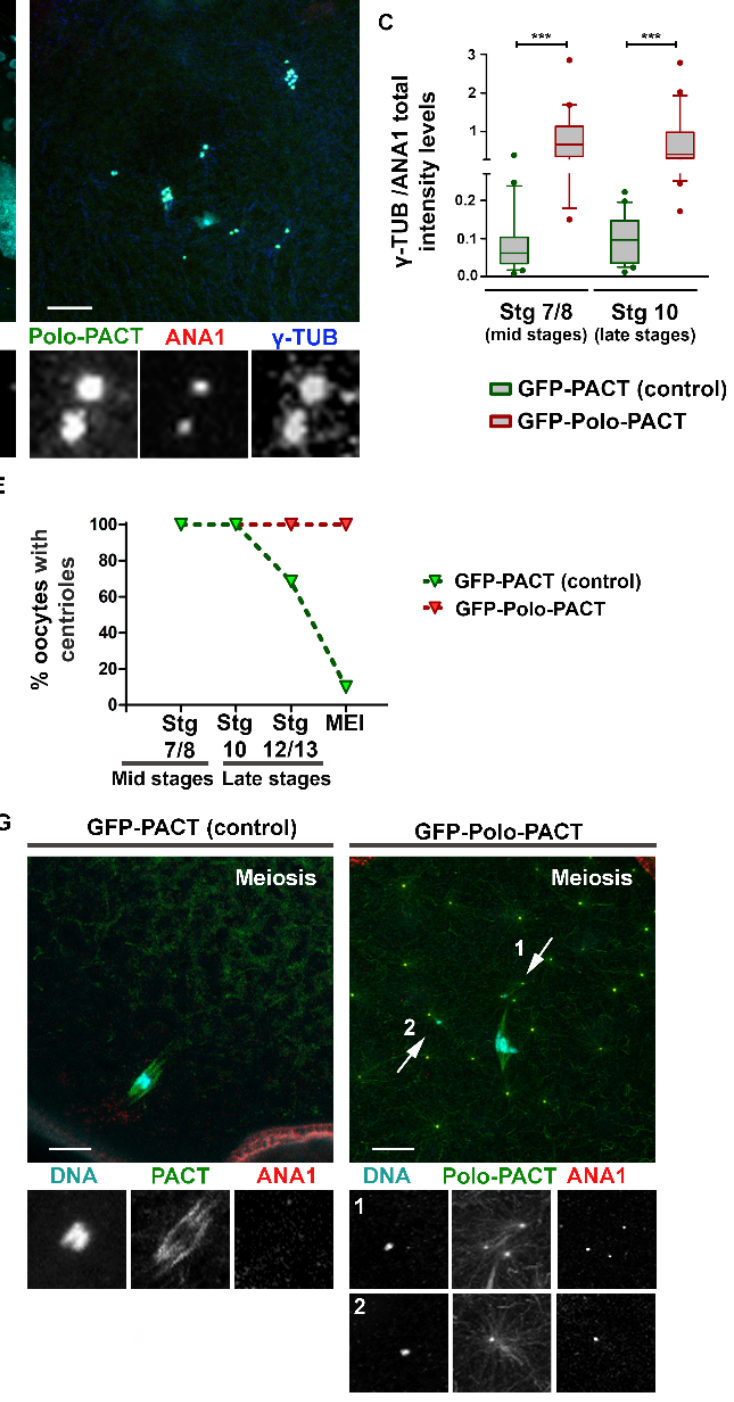

Fig. 3. Ectopic tethering of Polo to the centrioles prevents $P C M$ and centriole loss, leading to abnormal meiotic division. (A) Targeting Polo to the centrioles. GFP-PACT (control) and GFPPolo-PACT were expressed in the germline (PACT is the centriolar targeting domain of PLP \{Gard, 
1995 \#44\}). ANA1-tdTomato expression under endogenous promoter was used as a robust centriolar marker, and immunostaining for $\gamma$-tubulin ( $\gamma$-TUB) as a PCM marker. Enlargements of the indicated areas (arrows) are shown (8.5x and $5 \mathrm{x}$ magnification in $\mathrm{mid} /$ late stages and stages $12 / 13$, respectively, so that the images are set to comparable scales). All images were acquired with same exposure. Scale bars, $10 \mu \mathrm{m}$. (B) Quantification of oocytes showing $\gamma$-TUB at the oocyte's MTOC (co-localization between $\gamma$-TUB, ANA1 and PACT). Note that expression of GFPPolo-PACT retains $\gamma$-TUB at the MTOC'C. Dashed lines are included to help infer general trends (C) Quantification of the amount of $y$-TUB per ANA1 at the centrioles. Expression of GFP-Polo-PACT led to approximately $3 x$ more $y$-TUB on centrioles. A minimum of 30 oocytes were analyzed per stage in $B$ and $C$. The statistical difference between samples was evaluated with a t-test $(* * *$, $p<0.001$ ). (D) Quantification of total ANA1-tdTomato levels (endogenous promoter) in mid and late oogenesis stages in GFP-PACT and GFP-Polo-PACT expressing oocytes. Note that ANA1 levels are constant throughout oogenesis upon GFP-Polo-PACT expression suggesting that centrioles are maintained and that there is no centriole overduplication upon expression of Polo-PACT. Dashed lines are included to help infer general trends. More than 30 oocytes were scored per stage in $D$ and $E$. The statistical difference between samples was evaluated with a t-test ( $\left.{ }^{* *}, p<0.001\right)$. (E) Quantification of oocytes showing centrioles (oocytes with ANA1/total number of analyzed oocytes). Note that most meiosis I metaphases do not have centrioles in control oocytes (GFPPACT), while all analyzed oocytes expressing GFP-Polo-PACT show centrioles. Dashed lines are included to help infer general trends. (F-G) Different magnifications of stage 14 eggs expressing either GFP-PACT (control) or GFP-Polo-PACT. ANA1-tdTomato under the control of the endogenous promoter was used as a robust centriolar marker. F) Eggs expressing GFP-Polo-PACT show the maintenance of centrioles at the anterior of the egg in the vicinity of the meiotic DNA. Enlargements (5.8x magnification) of the indicated areas (arrows) are shown. Note that the nuclei surrounding the egg are from follicle cells. Scale bar, $40 \mu \mathrm{m}$. (G) Scattered DNA associates with maternal centrioles in meiosis in GFP-Polo-PACT-embryos. Enlargements (1.7x magnification) of the indicated areas (arrows) are shown. Scale bar, $10 \mu \mathrm{m}$. 
A
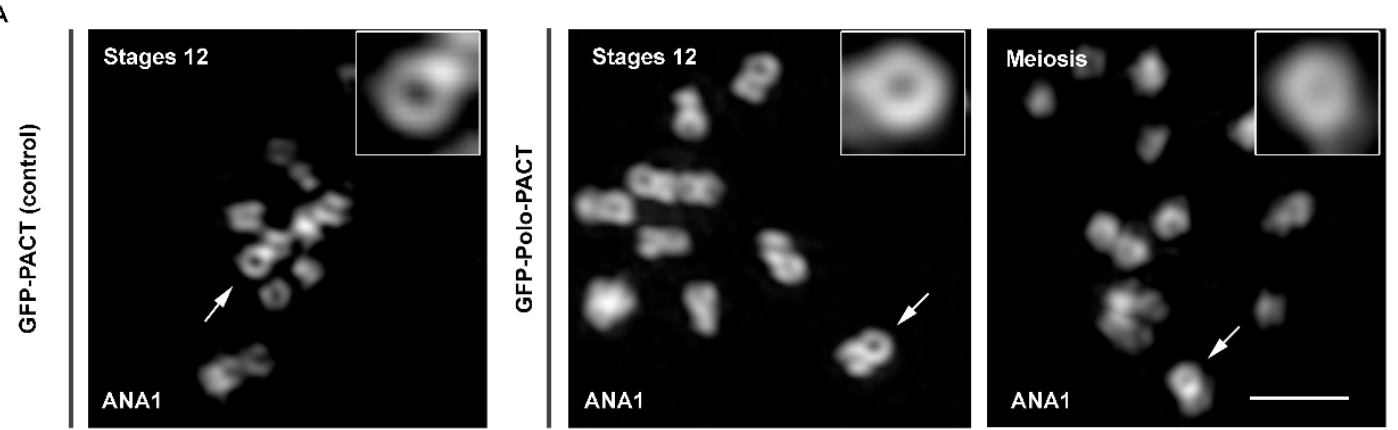

B
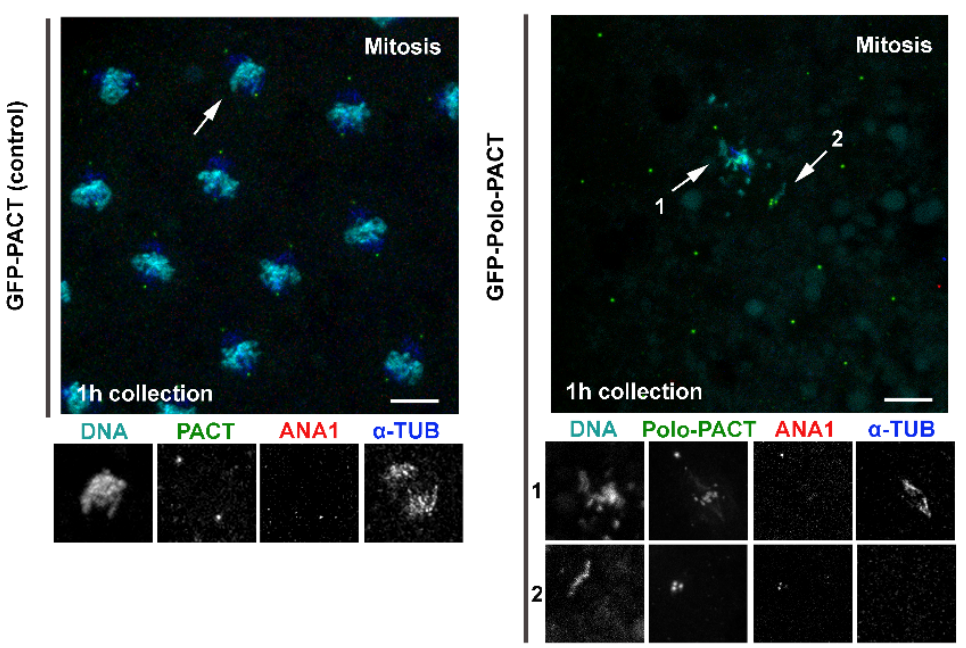

C

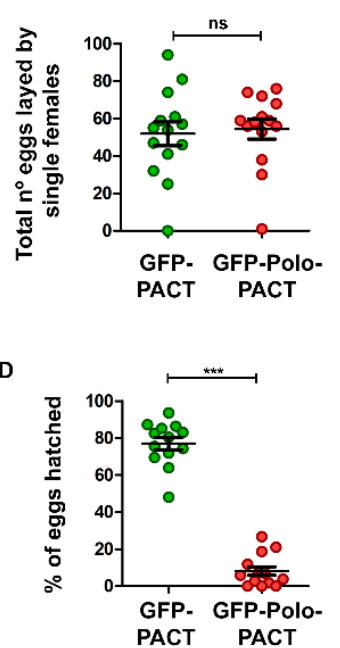

E

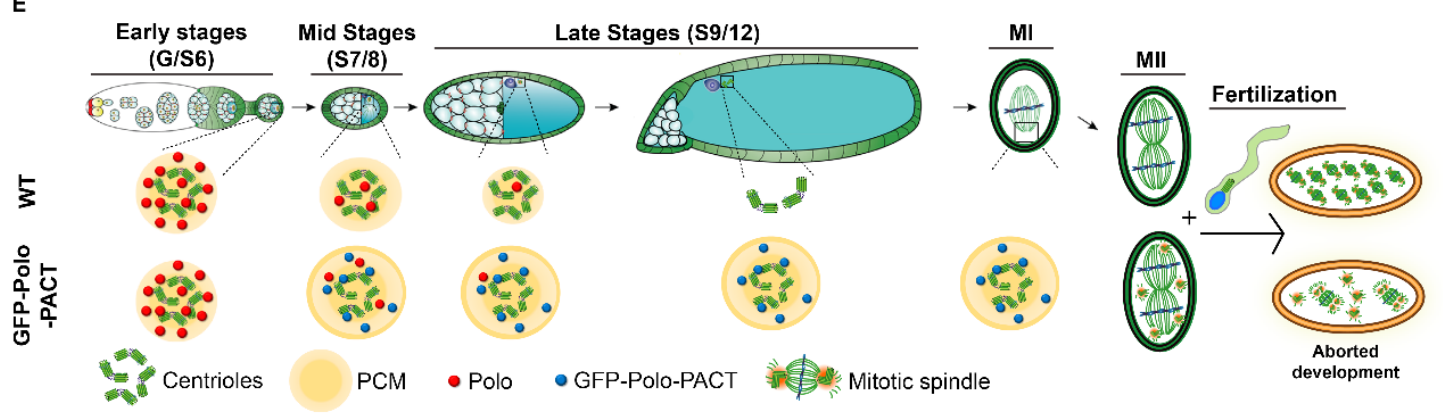

Fig. 4. Centrioles maintained in oogenesis show normal structure and lead to embryonic defects. (A) Representative super-resolution microscopy (Structured Illumination Microscopy, SIM) pictures of centrioles in eggs expressing either GFP-PACT (control) or GFP-Polo-PACT in stages 12 and in meiotic divisions. Centrioles are very small ( $250 \mathrm{~nm}$ across) and therefore their barrel like structure is only visible with super-resolution microscopy. ANA1-tdTomato expression under endogenous promoter was used to identify typical centriole barrel structures in stages 12 of both GFP-PACT and GFP-Polo-PACT expressing eggs. These structures were also present in meiotic divisions of GFP-Polo-PACT expressing eggs, but no structures were seen in control eggs at that stage (not shown), as expected from results using confocal microscopy (Fig. 1F and 3F,G). Similar barrel like structures were also seen in the green channel (GFP-PACT and GFP-Polo-PACT, not shown). Enlargements (4.3x magnification) of the indicated areas (arrows), focusing on a single centriole (cross section of the barrel), are shown. Scale bar $1 \mu \mathrm{m}$. (B) GFP-Polo-PACT embryos show several mitotic defects and mitotic arrest. Embryos were collected one hour after 
fertilization. GFP-Polo-PACT embryos show supernumerary centrioles and few divisions with scattered DNA associated with centrioles. The presence of centrioles in the embryos was analyzed by co-labeling with ANA1 (centriolar marker under endogenous promoter, very weak in early embryos and likely to be accumulated in older centrioles) and PACT. $\alpha$-tubulin ( $\alpha$-TUB, dark blue) was used to identify spindle shape. Enlargements (1.3x magnification) of the indicated areas (arrows) are shown. Scale bars, $10 \mu \mathrm{m}$. (C) Expression of GFP-Polo-PACT does not affect egg laying. Quantification of the number of eggs laid by GFP-PACT and GFP-Polo-PACT females (14 females per condition). (D) The majority of GFP-Polo-PACT fertilized eggs do not hatch. Quantification of hatched eggs in GFP-PACT and GFP-Polo-PACT. The statistical difference between samples in $C$ and $D$ was evaluated with a t-test $(* * *, p<0.001 ; n s$, non-significant). (E) Model of centriole elimination during oogenesis and the consequences of preventing Polo loss at the centrioles. Nearly all centrioles from the 16 cell cyst migrate to the oocyte in early stages of oogenesis. In a wild type fly, during oogenesis, there is both loss of expression and localization of Polo and PCM components from the oocytes centrioles, shutting down a centriole maintenance program. In the absence of PCM, the centrioles are not stable and are eliminated. Loss of centrioles in the female germline ensures their absence in meiosis, proper number of these structures upon fertilization and successful embryonic development. By expressing and anchoring Polo to the maternal centrioles, the maintenance program is "on", so the PCM is maintained in those structures and maternal centrioles are stable. Although the presence of active maternal centrioles does not preclude egg formation and fertilization, they lead to abnormal meiotic and mitotic divisions with consequently failed zygotic development. 